\title{
Ökonomische Effekte ausländischer Unternehmen in Deutschland
}

\begin{abstract}
Die Globalisierung ist mit komplexen Wertschöpfungsketten und den Wirtschaftstätigkeiten von Unternehmen verbunden, die von ausländischen Investoren kontrolliert werden. Welche ökonomischen Effekte haben ausländische Unternehmen auf das Bruttoinlandsprodukt und die Beschäftigung in Deutschland? Sie leisten einen Beitrag zur lokalen Wirtschaft, indem sie Arbeitskräfte beschäftigen, Waren und Dienstleistungen von lokalen Lieferanten kaufen und durch Spillover-Effekte die Produktivität lokaler Unternehmen steigern.
\end{abstract}

Maschinen, Handys, Computer - kaum ein Produkt wird mehr in nur einem Land hergestellt. Aufgrund der immer komplexeren globalen Wertschöpfungsketten überschreiten Waren und Zwischenprodukte mehrfach Grenzen, bevor sie ihre Endverbraucher erreichen. Multinationale Unternehmen spielen in diesem Prozess eine Schlüsselrolle, indem sie Fabriken, Vertriebsgesellschaften oder Forschungs- und Entwicklungszentren in einem anderen Land errichten und in lokale Unternehmen investieren oder diese erwerben. ${ }^{1}$

Multinationale Unternehmen investieren im Ausland, weil sie langfristig einen höheren Gewinn und Unternehmenswert erwarten. Ihre Aktivitäten können aber auch den wirtschaftlichen Wohlstand im Gastland erhöhen. Ein positiver Nettoeffekt ist jedoch nicht selbstverständlich, da neue Wirtschaftstätigkeiten ausländischer Unternehmen die Wirtschaftstätigkeiten lokaler Unternehmen verdrängen und Engpässe in der lokalen Wirtschaft verursachen können.

\section{Quantifizierung der ökonomischen Effekte}

Die Analyse greift auf Eurostat-Statistiken über die Struktur und Tätigkeit von Auslandsunternehmenseinheiten (Foreign Affiliates Statistics, FATS) zurück. ${ }^{2}$ Die FATS de-

(c) Der/die Autor(en) 2020. Open Access: Dieser Artikel wird unter der Creative Commons Namensnennung 4.0 International Lizenz (https:// creativecommons.org/licenses/by/4.0/deed.de) veröffentlicht.

Open Access wird durch die ZBW - Leibniz-Informationszentrum Wirtschaft gefördert.

1 Vgl. C. Jungbluth: Direktinvestitionen: Internationale Arbeitsteilung in einer globalisierten Welt, 26.7.2018, https://www.bertelsmann-stiftung.de/de/unsere-projekte/global-economic-dynamics/projektthemen/direktinvestitionen (17.10.2019).

2 Vgl. Bertelsmann Stiftung (Hrsg.): The Impact of Foreign-owned Firms in the EU and Germany, Diskussionspapier, Gütersloh 2020 (im Erscheinen). Hier werden neben den Effekten auf Deutschland auch die aggregierten Effekte auf die EU quantifiziert. finieren ein ausländisches Unternehmen als „ein im Land ansässiges Unternehmen, das von einer nicht im Land ansässigen institutionellen Einheit kontrolliert wird."3 Die FATS konzentrieren sich auf die ausländischen Unternehmen, die sich mehrheitlich im Besitz eines einzelnen Investors oder einer Gruppe verbundener Investoren befinden, die gemeinsam handeln und in der Regel mehr als $50 \%$ der Stammaktien oder der Stimmrechte besitzen. ${ }^{4}$

Unternehmen in mehrheitlich ausländischem Besitz beeinflussen die Beschäftigung und das Bruttoinlandspro-

3 „Kontrollierend ist hierbei die institutionelle Einheit, die letztlich die Kontrolle über eine Auslandsunternehmenseinheit ausübt. Es handelt sich um die institutionelle Einheit in der Kette der eine Auslandsunternehmenseinheit kontrollierenden Einheiten, die nicht von einer anderen institutionellen Einheit kontrolliert wird." Eurostat: Auslandsunternehmenseinheiten, https://ec.europa.eu/eurostat/de/web/structuralbusiness-statistics/global-value-chains/foreign-affiliates (16.10.2019).

4 Eurostat: Foreign Affiliates Statistics (FATS), Recommendations Manual, Luxemburg, 2012, S. 13, https://ec.europa.eu/eurostat/documents/3859598/5922981/KS-RA-12-016-EN.PDF/c93cdf48-5efa459f-b218-731a9a5476e9 (16.10.2019).

Dr. Cora Jungbluth ist Senior Expert International Trade and Investment bei der Bertelsmann Stiftung in Gütersloh.

Dr. Eva Rytter Sunesen ist Managing Economist bei
Copenhagen Economics in Kopenhagen.

Morten May Hansen, M. Sc., ist dort Economist.

Joshua Brown, M. Sc., ist dort Analyst. 
dukt (BIP) in ihrem Gastland. Dies kann über vier Transmissionskanäle erfolgen:

1. direkte Effekte, die die ausländischen Unternehmen selbst verursachen;

2. indirekte Effekte, die über lokale Unternehmen in der Lieferkette der ausländischen Unternehmen entstehen;

3. induzierte Effekte, die über den Konsum der direkt und indirekt, also innerhalb der Lieferketten, von in ausländischen Unternehmen Beschäftigten, entstehen;

4. Spillover-Effekte, die in lokalen Unternehmen entstehen.

Die Quantifizierung dieser Effekte basiert auf den folgenden drei Annahmen, die insgesamt konservative Ergebnisse erwarten lassen:

1. Das ausländische Unternehmen hat die gleiche Produktivität wie die durchschnittliche Firma in der gleichen Branche. Diese Annahme dürfte die Effekte ausländischer Unternehmen auf das BIP eher unterschätzen, da diese in der Regel produktiver sind als inländische Unternehmen.

2. Das ausländische Unternehmen hat das gleiche Kaufverhalten wie die durchschnittliche Firma in der gleichen Branche. Diese Annahme dürfte die Effekte der indirekten und induzierten Effekte leicht überschätzen. Der Grund dafür ist, dass ausländische Unternehmen eher in internationale Lieferketten eingebunden sind als inländische Unternehmen. Dies ist jedoch nur für die Lieferkette außerhalb der EU relevant. Lieferketten innerhalb der EU werden durch das Modell abgedeckt.

3. Die Arbeitnehmer, die direkt oder indirekt von ausländischen Unternehmen beschäftigt werden, weisen das gleiche Konsumverhalten auf wie die übrige Bevölkerung. Diese Annahme sollte keinen großen Einfluss auf die induzierten Effekte haben. Und wenn dies doch der Fall ist, ist der Unterschied nicht eindeutig. Denn wenn die direkt und indirekt in ausländischen Unternehmen Beschäftigen dazu neigen sollten, ihre Löhne anders auszugeben, würden die induzierten Effekte einfach in anderen Sektoren unterstützt werden, wenn auch nicht in der exakt gleichen Größenordnung.

\section{Direkte Effekte}

Die Zahl der Beschäftigten in ausländischen Unternehmen in Deutschland gemäß Eurostat dient als Ausgangspunkt zur Quantifizierung des direkten Effekts auf das BIP.
Diese Angaben entsprechen dem direkten Effekt ausländischer Unternehmen auf die Beschäftigung in ihrem Gastland. Im nächsten Schritt werden die direkten Effekte auf das BIP quantifiziert. Dazu verwendet Copenhagen Economics eine Input-Output-Tabelle aus der World Input-Output Database (WIOD), die Informationen über den BIP-Beitrag für jeden Sektor liefert. Darüber hinaus liegen von Eurostat Angaben zur sektoralen Beschäftigung vor. Zur Berechnung der direkten Effekte auf das BIP wird die Beschäftigung in ausländischen Unternehmen mit dem durchschnittlichen BIP-Beitrag ${ }^{5}$ pro Beschäftigten in jedem Sektor multipliziert:

$$
\frac{\text { BIP in Sektor i }}{\text { Beschäftigung in Sektor i }} \text { * } \begin{aligned}
& \text { Beschäftigung in ausländischen } \\
& \text { Firmen in Sektor i }
\end{aligned}
$$

Die Effekte auf Beschäftigung und BIP werden für 2014 gemessen, dem letzten Jahr, das derzeit in den WIOD-Tabellen verfügbar ist, und für 2014 bis 2017 analog zur Entwicklung des BIP und der Beschäftigung projiziert. Der Projektion unterliegt implizit die Annahme, dass BIP und Beschäftigung in den ausländischen Unternehmen und ihren Wertschöpfungsketten im gleichen Maße gewachsen sind wie in der übrigen Wirtschaft. Das Ergebnis dürfte konservativ ausfallen, da ausländische Unternehmen in der Regel sehr produktiv sind und daher stärker wachsen können als der Durchschnitt.

\section{Indirekte Effekte}

Die indirekten Effekte ergeben sich, wenn ausländische Unternehmen Waren und Dienstleistungen bei lokalen Lieferanten und diese wiederum Inputs von ihren lokalen Lieferanten kaufen und so weiter. Diese Effekte erstrecken sich auf die gesamte Lieferkette der EU und wirken sich indirekt auf Beschäftigung und BIP aus.

Für die Quantifizierung der indirekten Effekte kommt ein Multiplikatormodell zum Einsatz, das ebenfalls auf den WIOD-Tabellen basiert. Die indirekten Effekte können „pro direktem Arbeitsplatz“ oder „pro direktem BIP-Beitrag (z.B. in Euro)" gemessen werden. Dies sind die sogenannten Multiplikatoren. Das Modell kalkuliert so die Effekte auf Beschäftigung und BIP, die in den Lieferunternehmen des ausländischen Unternehmens entstehen.

\section{Induzierte Effekte}

Induzierte Effekte entstehen, wenn die direkt und indirekt von ausländischen Unternehmen Beschäftigten ihre

5 Bruttoinlandsprodukt zu Faktorkosten: Der BIP-Beitrag umfasst die Wertschöpfung und die Produktionsteuern. Potenzielle Subventionen werden abgezogen. 
Löhne und Gehälter ausgeben. Copenhagen Economics quantifiziert die induzierten Effekte mit dem gleichen Modell wie die indirekten Effekte und kalkuliert so die Effekte auf BIP und Beschäftigung, die sich aus dem Konsum der direkt und indirekt von ausländischen Unternehmen Beschäftigten ergeben.

\section{Spillover-Effekte}

Ausländische Unternehmen können auch durch sogenannte Spillover-Effekte auf inr Gastland einwirken. Dazu zählen Wissenstransfer (z.B. höhere Mobilität von Fachkräften), verstärkter Wettbewerb und vertikale Verbindungen in lokalen Lieferketten. Einige dieser Spillover-Effekte können positive oder negative Auswirkungen auf lokale Unternehmen haben. Wenn das ausländische Unternehmen beispielsweise alle seine Inputs von Lieferanten auBerhalb des lokalen Marktes bezieht und gleichzeitig lokale Wettbewerber verdrängt, die lokale Inputs einkaufen, reduziert sich die Produktivität unter lokalen Lieferanten aufgrund fehlender Größenvorteile.

Die Spillover-Effekte werden auf Basis einer ökonometrischen Regression geschätzt. ${ }^{6}$ Die Schätzungen basieren auf Unternehmensdaten aus dem Jahr 2015 für ausländische und inländische Unternehmen in 34 Ländern Europas. Die Fortschreibung der Ergebnisse bis 2017 erfolgt analog zu den übrigen Effekt-Gruppen. Mithilfe dieser Daten schätzt Copenhagen Economics die Auswirkungen einer Zunahme der Konzentration ausländischer Unternehmen auf die Arbeitsproduktivität, approximiert durch Umsatz pro Mitarbeiterin, in den lokalen Unternehmen in derselben Branche und Region (branchenspezifische Spillover) bzw. derselben Region, unabhängig von der Branche (weitergehende regionale Spillover).

Das Modell für die Berechnung industriespezifischer Spillover-Effekte sieht wie folgt aus:

In Arbeitsproduktivität ${ }_{i \mathrm{jk}}=\mathrm{f}\left(\mathrm{FDI}\right.$ Konzentration $_{\mathrm{ji}}$, In Kapitalintensität ${ }_{i \mathrm{jk}}$, Alter ${ }_{\mathrm{ijk}}$, Alter $_{\mathrm{ijk}}{ }^{2}$, Region/Branchengröße $_{\mathrm{ij}}$, Wachstumsrate BIP pro Kopf )

Der natürliche Logarithmus der Arbeitsproduktivität eines bestimmten Unternehmens $(k)$ in einer bestimmten zweistelligen NACE-Industrie ${ }^{7}(\mathrm{j})$ in einer bestimmten NUTS3-

6 Für eine detaillierte methodologische Beschreibung vgl. Copenhagen Economics: The World in Europe, global FDI Flows towards Europe Impacts of extra-European FDI towards Europe, März 2018, https:// www.espon.eu/sites/default/files/attachments/ESPON\%20FDI\%20 -\%2006\%20-\%20Scientific\%20report\%20-\%20Impacts\%20of\%20 extra-European\%20FDI.pdf (17.10.2019).

$7 \mathrm{NACE}=$ Nomenclature statistique des activités économiques dans la Communauté européenne (Statistische Systematik der Wirtschaftszweige in der Europäischen Gemeinschaft).
Region $^{8}$ (i) wird hier als Funktion der Konzentration von ausländischen Unternehmen in der betreffenden Branche und Region modelliert. Die Konzentration ausländischer Unternehmen wird als der Anteil aller Beschäftigten gemessen, die in ausländischen Unternehmen innerhalb der jeweiligen Branche und Region arbeiten.

Das Modell, das weitergehende regionale Spillover-Effekte schätzt, sieht ähnlich aus. Da der Schwerpunkt jedoch nun auf der Identifizierung der Spillover-Effekte ausländischer Unternehmen in allen Branchen innerhalb einer bestimmten NUTS3-Region liegt, wird die Konzentration ausländischer Unternehmen auf regionaler Ebene als der Anteil aller Mitarbeiter gemessen, die in einem ausländischen Unternehmen innerhalb einer bestimmten Region arbeiten.

In beiden Fällen wird für mehrere andere unternehmensspezifische, branchenspezifische und regionale Faktoren kontrolliert, die sich ebenfalls auf die Produktivität lokaler Unternehmen auswirken können. Das Modell beinhaltet Dummy-Variablen für Länder und für NACE2. Die Methodik und die verwendeten Kontrollvariablen wurden nach einer Überprüfung der vorhandenen empirischen Literatur zu Spillover-Effekten ausgewählt.. ${ }^{9}$ Zu den Dummy-Variablen gehören beispielsweise Kapitalintensität, BIP-Wachstum pro Kopf, Größe der Region und der Industrie usw. Die Spillover-Effekte werden auf EU-Ebene quantifiziert, um genügend Beobachtungen zur Berechnung der Auswirkungen zu haben. Für Deutschland allein ist das nicht möglich, da es zu wenige Beobachtungen gibt. Daher werden die EU-Spillover auf sektoraler Basis für die Berechnung der Spillover-Effekte in Deutschland verwendet. Das bedeutet, dass detaillierte Informationen über die Konzentration ausländischer Unternehmen auf sektoraler Basis in Deutschland verwendet werden, aber davon ausgegangen wird, dass die Produktivitätssteigerungen in deutschen Unternehmen dem EU-Durchschnitt in derselben Branche entsprechen.

\section{Effekte ausländischer Unternehmen auf Beschäftigung und BIP in Deutschland}

2017 gab es in Deutschland der Projektion von Copenhagen Economics nach rund 33000 ausländische Unternehmen. Die Zahl der dort direkt Beschäftigten belief sich auf 3,4 Mio. Das sind $8 \%$ der Erwerbstätigen. Der Anteil steigt auf $33 \%$ oder fast 15 Mio. Arbeitsplätze, wenn man die indirekten und induzierten Effekte berücksichtigt. Fast die Hälfte dieser Arbeitsplätze (7 Mio.) wurde durch den

8 NUTS $=$ Nomenclature des unités territoriales statistiques (Statistische Systematik der Gebietseinheiten).

9 Vgl. Copenhagen Economics, a.a. O. 


\section{Abbildung 1}

Beschäftigungseffekte ausländischer Unternehmen in Deutschland, 2017

in Mio. Arbeitsplätzen

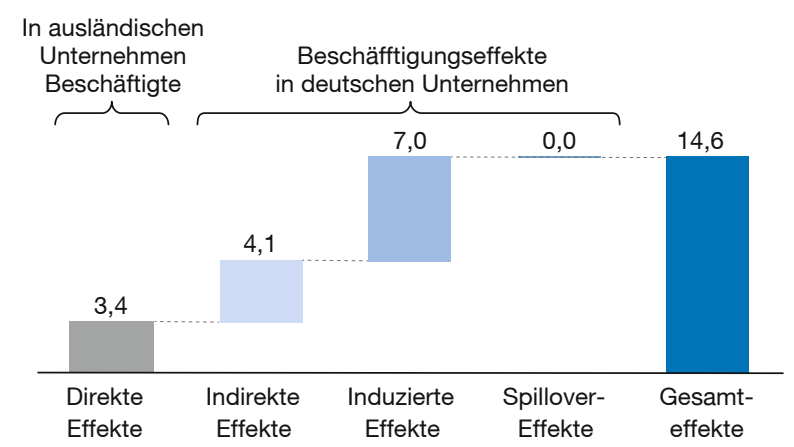

Anmerkung: Die Berechnungen basieren auf FATS-Daten von Eurostat. Die Ergebnisse wurden analog zur Entwicklung der Beschäftigung in Deutschland von 2014 bis 2017 projiziert. Die Effekte sind gerundet. Der exakte Gesamteffekt beträgt 14,56 Mio. Arbeitsplätze.

Quelle: Copenhagen Economics, basierend auf Eurostat und WIOD.

privaten Konsum der direkt und indirekt in den ausländischen Unternehmen Beschäftigten geschaffen (vgl. Abbildung 1).

Die ökonometrische Analyse ergibt keine Spillover-Effekte auf die Beschäftigung (siehe auch Kasten 1). Das deutet darauf hin, dass sich die positiven und negativen Auswirkungen, die ausländische Unternehmen auf die Beschäftigung in lokalen Unternehmen haben, z. B. durch Produktivitätssteigerungen, eine erhöhte Nachfrage nach lokal produzierten Waren und Dienstleistungen oder durch Wettbewerbseffekte, neutralisieren. Hinzu kommt die Annahme, dass ausländische Unternehmen auf lange Sicht, d.h. 30 Jahre, keine Effekte auf die Beschäftigung haben. Wenn es keine ausländischen Unternehmen gäbe, würden dennoch Arbeitsplätze durch inländische Investoren geschaffen. Aber der Prozess könnte länger dauern und kurzfristig zu mehr Arbeitslosigkeit führen.

2017 betrug das direkt von ausländischen Unternehmen in Deutschland erwirtschaftete BIP 300 Mrd. Euro (9\% des deutschen BIP). Bezieht man indirekte und induzierte Effekte ein, haben ausländische Unternehmen 2017 fast 1100 Mrd. Euro oder $33 \%$ des deutschen BIP unterstützt (vgl. Abbildung 2). Den gleichen Anteil leisten sie auch für die Beschäftigung. Das deutet darauf hin, dass die Produktivität der von ausländischen Unternehmen bewirkten Beschäftigung der durchschnittlichen Produktivität der Gesamtbeschäftigung entspricht. Für das BIP lassen sich auch Spillover-Effekte beobachten: Diese entstehen, weil ausländische Unternehmen die Produktivität lokaler Un-

\author{
Abbildung 2 \\ BIP-Effekte ausländischer Unternehmen in \\ Deutschland, 2017 \\ in Mrd. Euro
}

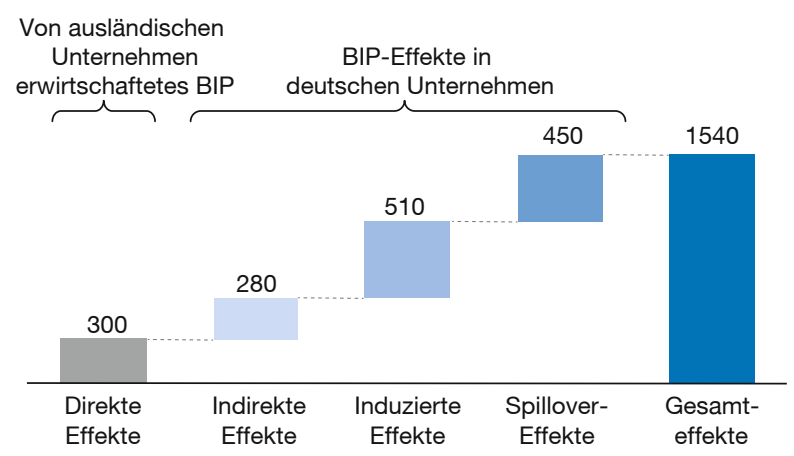

Anmerkung: Die Berechnungen basieren auf FATS-Daten von Eurostat. Die Ergebnisse wurden analog zur Entwicklung des deutschen BIP von 2014 bis 2017 projiziert.

Quelle: Copenhagen Economics, basierend auf Eurostat und WIOD.

ternehmen steigern und so deren Wettbewerbsfähigkeit erhöhen. Für 2017 ergaben sich Spillover-Effekte in Höhe von 450 Mrd. Euro. Der Gesamteffekt ausländischer Unternehmen auf das deutsche BIP belief sich 2017 somit auf 1540 Mrd. Euro oder $47 \%$ des deutschen BIP.

\section{Fazit und Empfehlungen}

Die Berechnungen von Copenhagen Economics zeigen, dass die Wirtschaftsaktivitäten von Unternehmen in mehrheitlich ausländischem Besitz einen positiven direkten Effekt auf Beschäftigung und BIP in Deutschland haben. Zudem erhöhen sie die Nachfrage nach Waren und Dienstleistungen durch indirekte und induzierte Effekte, was den Beitrag zu Beschäftigung und BIP lokaler Unternehmen erhöht. Durch Spillover-Effekte können ausländische Unternehmen auch die Produktivität und das Wachstum in lokalen Unternehmen steigern, z. B. durch Wissenstransfer und verstärkten Wettbewerb. Die Betrachtung dieser vier Effektgruppen ermöglicht es erst, die Bedeutung ausländischer Unternehmen für ihr Gastland umfassender zu bewerten.

Jenseits der quantitativen Effekte können ausländische Unternehmen zudem die Vernetzung des Gastlandes mit den internationalen Märkten verbessern, z.B. durch die Intensivierung von Wertschöpfungsketten und einen besseren, da direkten, Kundenzugang. Das könnte sich im Umgang mit den beiden Megatrends Digitalisierung und demografischer Wandel als nützlich erweisen: Sollten sich additive Produktionstechniken (3D-Druck) verbrei- 
ten, werden die Waren zunehmend dort produziert, wo sie konsumiert werden. Dies erfordert den Aufbau lokaler Produktionskapazitäten, d.h. ein zunehmendes strategisch und langfristig ausgerichtetes Engagement ausländischer Unternehmen in Form von Direktinvestitionen vor Ort. Der traditionelle Außenhandel könnte in Zukunft also zurückgehen, während Investitionen von Unternehmen aus dem Ausland zunehmen dürften. ${ }^{10}$ Wenn Deutschland für ausländische Unternehmen attraktiv bleibt, könnten diese zudem dazu beitragen, den demografischen Wandel abzumildern. Da dieser in Zukunft einen Rückgang der inländischen Investitionen zur Folge haben könnte, könnten Investitionen ausländischer Unternehmen diese Lücke teilweise schließen und langfristig das BIP und den Kapitalstock in einer alternden Gesellschaft erhöhen. ${ }^{11}$

Aus Sicht der Bertelsmann Stiftung ergeben sich daraus drei Vorschläge, wie sich der künftige Umgang mit Direktinvestitionen ausländischer Unternehmen wirtschaftspolitisch gestalten könnte:

1. Ausbau der Kommunikation hinsichtlich der ökonomischen Bedeutung ausländischer Unternehmen für den Wirtschaftsstandort Deutschland: In Zeiten zunehmenden Protektionismus und Populismus ist es wichtiger denn je, auf die Bedeutung global vernetzter Märkte und Wertschöpfungsketten hinzuweisen.

2. Verbesserung der nationalen Rahmenbedingungen für Direktinvestitionen ausländischer Investoren bei gleichzeitiger Vermeidung eines „Race to the Bottom“ (z. B. ungesunder Steuerwettbewerb oder Absenkung der Arbeits-, Sozial- und Umweltstandards): Konkret könnte z.B. die unterstützende Rolle der deutschen Stand-

10 Vgl. T. Petersen: How 3D Printing Technology Could Change World Trade: Five Predictions on the Future of Global Trade, 2019, https:// ged-project.de/allgemein-en/how-3d-printing-technology-couldchange-world-trade/ (17.10.2019).

11 Vgl. A. Esche, M. Lizarazo López, T. Petersen: Fostering Productivity - Investment and Demographic Transition, T20 Policy Brief, 2019, https://t20japan.org/wp-content/uploads/2019/04/t20-japantf10-2-fostering-prosperity-investment-demographic-transition.pdf (17.10.2019)

\section{Kasten 1 \\ Beispiele für Spillover-Effekte}

Spillover-Effekte auf die Beschäftigung: Die ökonometrische Analyse ergibt keine Spillover-Effekte auf die Beschäftigung. Während ausländische Unternehmen durch die indirekten und induzierten Effekte Beschäftigung in lokalen Unternehmen unterstützen, manifestieren sich die produktivitätssteigernden Effekte im Durchschnitt nicht in Form einer erhöhten Beschäftigung in den lokalen Unternehmen. Negative Effekte kompensieren positive Effekte und neutralisieren den Spillover-Effekt auf die Beschäftigung.

Spillover-Effekte auf das BIP: Die ökonometrische Analyse zeigt jedoch, dass ausländische Unternehmen die Produktivität in inländischen Unternehmen positiv beeinflussen. Das ausländische Unternehmen bringt z. B. Wissen über Produkte, Lieferketten und Technologien mit, das in Form einer höheren Produktivität auf lokale Unternehmen „übergreift“. Daraus ergibt sich ein BIP-steigernder Spillover-Effekt.

ortmarketingagentur Germany Trade and Invest (GTAl) für ausländische Investoren gestärkt werden.

3. Verbesserung der internationalen Rahmenbedingungen für ausländische Direktinvestitionen: Gegenwärtig werden diese hauptsächlich in bilateralen Investitionsabkommen oder Investitionskapiteln in Freihandelsabkommen geregelt, d.h., die bestehenden Regularien sind stark fragmentiert. Langfristig wäre daher ein multilateraler Ansatz für grenzüberschreitende Investitionen sinnvoll - auch wenn ein solcher vor dem Hintergrund der gegenwärtigen Krise der Welthandelsorganisation (WTO) als Inbegriff des Multilateralismus zunächst noch ehrgeizig erscheint. Deutschland sollte daher bestehende Initiativen zur Erleichterung von grenzüberschreitenden Investitionen innerhalb der G20 und der WTO aktiv unterstützen.

Title: Economic Effects of Foreign Companies in Germany

Abstract: Foreign-owned firms play an important role in global value chains and can positively impact their host economy. A positive net effect should not be taken for granted, though, as new economic activities of foreign-owned firms can crowd out economic activity of local companies. The analysis shows that the overall effect of foreign-owned firms on the German economy is positive. From an economic policy perspective, it would make sense to better communicate the benefits of foreign-owned firms and improve national and international framework conditions for foreign investors while at the same time avoiding a 'race to the bottom' such as unhealthy tax competition.

JEL Classification: F20, F21, F23 